\title{
TU/e EmonOWEN

\section{Impact of the Peterlin approximation on polymer dynamics in turbulent flows}

\section{Citation for published version (APA):}

Vincenzi, D., Perlekar, P., Biferale, L., \& Toschi, F. (2015). Impact of the Peterlin approximation on polymer dynamics in turbulent flows. Physical Review E - Statistical, Nonlinear, and Soft Matter Physics, 92(5), [053004]. https://doi.org/10.1103/PhysRevE.92.053004

DOI:

10.1103/PhysRevE.92.053004

Document status and date:

Published: 05/11/2015

\section{Document Version:}

Publisher's PDF, also known as Version of Record (includes final page, issue and volume numbers)

\section{Please check the document version of this publication:}

- A submitted manuscript is the version of the article upon submission and before peer-review. There can be important differences between the submitted version and the official published version of record. People interested in the research are advised to contact the author for the final version of the publication, or visit the $\mathrm{DOI}$ to the publisher's website.

- The final author version and the galley proof are versions of the publication after peer review.

- The final published version features the final layout of the paper including the volume, issue and page numbers.

Link to publication

\section{General rights}

Copyright and moral rights for the publications made accessible in the public portal are retained by the authors and/or other copyright owners and it is a condition of accessing publications that users recognise and abide by the legal requirements associated with these rights.

- Users may download and print one copy of any publication from the public portal for the purpose of private study or research.

- You may not further distribute the material or use it for any profit-making activity or commercial gain

- You may freely distribute the URL identifying the publication in the public portal.

If the publication is distributed under the terms of Article 25fa of the Dutch Copyright Act, indicated by the "Taverne" license above, please follow below link for the End User Agreement:

www.tue.nl/taverne

Take down policy

If you believe that this document breaches copyright please contact us at:

openaccess@tue.nl

providing details and we will investigate your claim. 


\title{
Impact of the Peterlin approximation on polymer dynamics in turbulent flows
}

\author{
Dario Vincenzi, ${ }^{1}$ Prasad Perlekar, ${ }^{2}$ Luca Biferale, ${ }^{3}$ and Federico Toschi ${ }^{4,5}$ \\ ${ }^{1}$ Laboratoire Jean Alexandre Dieudonné, Université Nice Sophia Antipolis, CNRS, UMR 7351, 06100 Nice, France \\ ${ }^{2}$ TIFR Center for Interdisciplinary Sciences, Tata Institute of Fundamental Research, Narsingi, Hyderabad 500075, India \\ ${ }^{3}$ Department of Physics and INFN, University of Rome "Tor Vergata," Via della Ricerca Scientifica 1, 00133 Roma, Italy \\ ${ }^{4}$ Department of Applied Physics, and Department of Mathematics and Computer Science, Eindhoven University of Technology, \\ 5600 MB Eindhoven, The Netherlands \\ ${ }^{5}$ IAC, CNR, Via dei Taurini 19, I-00185 Roma, Italy
}

(Received 3 June 2015; revised manuscript received 31 August 2015; published 5 November 2015)

\begin{abstract}
We study the impact of the Peterlin approximation on the statistics of the end-to-end separation of polymers in a turbulent flow. The finitely extensible nonlinear elastic (FENE) model and the FENE model with the Peterlin approximation (FENE-P) are numerically integrated along a large number of Lagrangian trajectories resulting from a direct numerical simulation of three-dimensional homogeneous isotropic turbulence. Although the FENE-P model yields results in qualitative agreement with those of the FENE model, quantitative differences emerge. The steady-state probability of large extensions is overestimated by the FENE-P model. The alignment of polymers with the eigenvectors of the rate-of-strain tensor and with the direction of vorticity is weaker when the Peterlin approximation is used. At large Weissenberg numbers, the correlation times of both the extension and of the orientation of polymers are underestimated by the FENE-P model.
\end{abstract}

DOI: 10.1103/PhysRevE.92.053004

PACS number(s): 47.27.Gs, 47.57.Ng

\section{INTRODUCTION}

The addition of elastic polymers to a Newtonian solvent introduces a history dependence in the response of the fluid to a deformation and hence modifies the rheological properties of the solvent [1]. In turbulent flows, the non-Newtonian nature of polymer solutions manifests itself through a considerable reduction of the turbulent drag compared to that of the solvent alone [2-6]. What renders this phenomenon even more remarkable is that an appreciable drag reduction can already be observed at very small polymer concentrations (of the order of a few parts per million). Turbulent drag reduction was discovered by Toms [7] more than 60 years ago and is now routinely used to reduce energy losses in crude-oil pipelines [8]. A full understanding of turbulent drag reduction remains nonetheless a difficult challenge, because in the turbulent flow of a polymer solution the dynamics of a large number of polymers is coupled with strongly nonlinear transfers of kinetic energy.

The study of turbulent flows of polymer solutions is essentially based on two approaches: the molecular approach and the continuum one. In the molecular (or Brownian dynamics) approach, a polymer is modeled as a sequence of $N$ beads connected by elastic springs. The deformation of the bead-spring chain is then followed along the trajectory of its center of mass. In homogeneous and isotropic turbulence, Watanabe and Gotoh [9] have shown that $N=2$ beads are in fact sufficient to describe the stationary statistics of both the extension and the orientation of polymers, i.e., the deformation of a polymeric chain is dominated by its slowest oscillation mode. An analogous conclusion has been reached by Terrapon et al. [10] in a study of polymer dynamics in a turbulent channel flow. The model consisting of only $N=2$ beads is known as the finitely extensible nonlinear elastic (FENE) dumbbell model [1]. The molecular approach is suitable for studying the deformation of passively transported polymers [9-21]. Molecular dynamics has also been employed in combination with standard Eulerian techniques to simulate viscoelastic flows (see the CONNFFESSIT $[22,23]$ and $\mathrm{BCF}$ [24] approaches). Peters and Schumacher [25] and Watanabe and Gotoh [26,27] have recently performed two-way coupling simulations of turbulent drag reduction in which the polymer feedback is given by the forces exerted on the fluid by a large number of individual dumbbells.

In practical applications, numerical simulations of turbulent flows of polymer solutions commonly use the continuum approach. The conformation of polymers is then described by means of a space- and time-dependent tensor field, which represents the average inertia tensor of polymers at a given time and position in the fluid. Such a tensor is termed the polymer conformation tensor. An evolution equation for the conformation tensor may be derived in principle from the FENE dumbbell model. Such an equation, however, involves the average over thermal fluctuations of a nonlinear function of the polymer end-to-end vector; a closure approximation is therefore required. Peterlin [28] proposed a mean-field closure according to which the average of the elastic force over thermal fluctuations is replaced by the value of the force at the mean-squared polymer extension. The resulting model was subsequently dubbed the FENE-P model [29]. Within the FENE-P model, the backreaction of polymers on the flow is described by a stress tensor field, which depends on the polymer conformation tensor. This continuum model is thus suitable for simulating turbulent flows of polymer solutions; it indeed amounts to simultaneously solving the evolution equation for the polymer conformation tensor and the NavierStokes equation with an additional elastic-stress term. The FENE-P model is widely employed in numerical simulations of turbulent drag reduction and has been successfully applied to channel flows [30-33], shear flows [34,35], and twoand three-dimensional homogeneous and isotropic turbulence [36-39]. Nevertheless, although it qualitatively reproduces the main features of turbulent drag reduction, the FENE-P model 
generally does not yield results in quantitative agreement with experimental data (e.g., Ref. [33]). It is therefore essential to assess the validity of the assumptions upon which the model is based.

For laminar flows, the Peterlin approximation has been examined in detail (see Refs. [40,41] and references therein). In particular, the FENE-P model is a good approximation of the FENE model in steady flows, whereas appreciable differences appear in time-dependent flows. This observation suggests that in turbulent flows, important differences between the two models should be expected. Several studies have subsequently investigated the validity of the Peterlin approximation in turbulent flows by comparing one-way coupling simulations of the FENE and FENE-P models [10,12,13,15,19]. These studies have clearly shown potential differences between the FENE and FENE-P models together with high sensitivity on the statistical ensemble and dependency on the degree of homogeneity of the underlying velocity fluctuations. We undertake a systematic analysis of the Peterlin approximation in three-dimensional homogeneous and isotropic turbulence by means of one-way coupling Lagrangian simulations of the FENE and FENE-P models. The size of our statistical ensemble $\left(128 \times 10^{3}\right.$ fluid trajectories and $2 \times 10^{3}$ realizations of thermal noise per trajectory) allows us to fully characterize the statistics of polymer extension and orientation and to investigate certain properties of the polymer conformation tensor that, to the best of our knowledge, had not been studied before. When the flow is turbulent, two independent effects are at the origin of the discrepancies between the FENE and FENE-P models: one is directly related to the closure approximation for the elastic force, while the other is of a statistical nature and is a consequence of deriving the statistics of the end-to-end vector from that of the conformation tensor. By isolating these two effects, we compare the steady-state statistics and the temporal correlation of the extension and of the orientation of polymers in the FENE and FENE-P models.

The rest of the paper is organized as follows. In Sec. II we briefly review the FENE and FENE-P models. The Lagrangian simulations are described in Sec. III. The results of the simulations are presented in Sec. IV. Finally, conclusions are drawn in Sec. V.

\section{FENE AND FENE-P MODELS}

In the FENE model, a polymer is described as two beads connected by an elastic spring, i.e., as an elastic dumbbell [1]. If the fluid is at rest, the polymer is in a coiled configuration because of entropic forces, and its equilibrium extension is determined by the intensity of thermal fluctuations. If the polymer is introduced in a moving fluid and the velocity field changes over the size of the polymer, then the polymer can stretch and deform. The dynamics of the polymer thus results from the interplay between the stretching action of the velocity gradient and the elastic force, which tends to take the polymer back to its equilibrium configuration.

The maximum extension of the dumbbell is assumed to be smaller than the Kolmogorov scale, so that the velocity field changes linearly in space at the scale of the dumbbell. The drag force on the beads is given by the Stokes law. Moreover, inertial effects and hydrodynamical interactions between the beads are disregarded. Polymer-polymer hydrodynamical interactions are also disregarded under the assumption that the polymer concentration is very low. Thus, the separation vector between the beads, $\boldsymbol{R}(t)$, satisfies the following stochastic ordinary differential equation (the FENE equation) $[1,42]$ :

$$
\frac{d \boldsymbol{R}}{d t}=\sigma(t) \boldsymbol{R}-\frac{\phi\left(R^{2}\right)}{2 \tau_{p}} \boldsymbol{R}+\sqrt{\frac{R_{0}^{2}}{\tau_{p}}} \boldsymbol{\xi}(t),
$$

where $R=|\boldsymbol{R}|, \sigma_{i j}(t)=\partial_{j} u_{i}(t)$ is the velocity gradient at the position of the center of mass of the dumbbell, $\boldsymbol{\xi}(t)$ is threedimensional white noise, $R_{0}$ is the polymer root-mean-square equilibrium extension, and $\tau_{p}$ is the polymer relaxation time [ $\tau_{p}$ is the time scale that describes the exponential relaxation of $\left\langle R^{2}(t)\right\rangle$ to its equilibrium value in the absence of flow]. The three terms on the right-hand side of Eq. (1) represent the stretching by the velocity gradient, the restoring elastic force, and thermal noise, respectively. The function $\phi$ determines the elastic force, and, in the FENE model, it has the following form:

$$
\phi(\zeta)=\frac{1}{1-\zeta / L^{2}}
$$

where $L$ is the maximum extension of the polymer. The elastic force diverges as $R$ approaches $L$, hence extensions greater than $L$ are forbidden. Note that $\boldsymbol{R}(t)$ is a random vector and that, when $\boldsymbol{u}$ is turbulent, two independent sources of randomness influence its evolution: thermal noise and the velocity gradient itself.

The polymer conformation tensor is defined as $\mathcal{C}_{i j} \equiv$ $\left\langle R_{i} R_{j}\right\rangle_{\xi}$, where $\langle\cdot\rangle_{\xi}$ denotes an average over thermal fluctuations. To derive the evolution equation for $\mathcal{C}$, we apply the Itô formula to $R_{i} R_{j}$ and use Eq. (1):

$$
\begin{aligned}
\frac{d}{d t}\left(R_{i} R_{j}\right)= & \frac{d R_{i}}{d t} R_{j}+R_{i} \frac{d R_{j}}{d t}+\frac{R_{0}^{2}}{\tau_{p}} \delta_{i j} \\
= & \sigma_{i k}(t) R_{k} R_{j}+\sigma_{j k}(t) R_{k} R_{i}-\frac{1}{\tau_{p}} \phi\left(R^{2}\right) R_{i} R_{j} \\
& +\frac{R_{0}^{2}}{\tau_{p}} \delta_{i j}+\sqrt{\frac{R_{0}^{2}}{\tau_{p}}}\left[R_{j} \xi_{i}(t)+R_{i} \xi_{j}(t)\right] .
\end{aligned}
$$

Whereas there is no Itô-Stratonovich ambiguity for Eq. (1), Eq. (3) should be understood in the Itô sense. We now average Eq. (3) with respect to the realizations of $\boldsymbol{\xi}(t)$ and make use of the following property of the Itô integral: $\left\langle R_{i} \xi_{j}(t)\right\rangle_{\xi}=0$. We thus obtain

$$
\begin{aligned}
\frac{d}{d t}\left\langle R_{i} R_{j}\right\rangle_{\xi}= & \sigma_{i k}(t)\left\langle R_{k} R_{j}\right\rangle_{\xi}+\sigma_{j k}(t)\left\langle R_{k} R_{i}\right\rangle_{\xi} \\
& -\frac{1}{\tau_{p}}\left[\left\langle\phi\left(R^{2}\right) R_{i} R_{j}\right\rangle_{\xi}-R_{0}^{2} \delta_{i j}\right] .
\end{aligned}
$$

Equation (4) is not closed with respect to $\mathcal{C}$ because of the term

$$
\mathcal{A}_{i j} \equiv\left\langle\phi\left(R^{2}\right) R_{i} R_{j}\right\rangle_{\xi} .
$$

To obtain a closed equation, Peterlin [28] proposed the following approximation:

$$
\mathcal{A}_{i j} \approx \phi\left(\left\langle R^{2}\right\rangle_{\xi}\right)\left\langle R_{i} R_{j}\right\rangle_{\xi}=\phi(\operatorname{tr} \mathcal{C}) \mathcal{C}_{i j}
$$


In the following, we shall denote

$$
\mathcal{T} \equiv \phi(\operatorname{tr} \mathcal{C}) \mathcal{C}
$$

The resulting evolution equation for the polymer conformation tensor (the FENE-P equation) is

$$
\frac{d \mathcal{C}^{\mathrm{P}}}{d t}=\sigma(t) \mathcal{C}^{\mathrm{P}}+\mathcal{C}^{\mathrm{P}} \sigma^{\mathrm{t}}(t)-\frac{1}{\tau_{p}}\left[\phi\left(\operatorname{tr} \mathcal{C}^{\mathrm{P}}\right) \mathcal{C}^{\mathrm{P}}-R_{0}^{2} \mathcal{I}\right]
$$

where $\mathcal{I}$ is the identity matrix and $\mathcal{C}^{\mathrm{P}}$ denotes the polymer conformation tensor calculated according to the Peterlin approximation. If the flow is turbulent, both $\mathcal{C}$ and $\mathcal{C}^{\mathrm{P}}$ have a random behavior. Equation (8) describes the evolution of the conformation tensor of a polymer along the Lagrangian trajectory of its center of mass. Numerical simulations of drag reduction use the Eulerian counterpart of Eq. (8), which is obtained by replacing $d \mathcal{C}^{\mathrm{P}} / d t$ with $\partial_{t} \mathcal{C}^{\mathrm{P}}+\boldsymbol{u} \cdot \nabla \mathcal{C}^{\mathrm{P}}$ and $\sigma(t)$ with the Eulerian velocity gradient. In principle, the evolution equation for the conformation tensor should be coupled with the Navier-Stokes equations through an additional stress term proportional to $\phi\left(\operatorname{tr} \mathcal{C}^{\mathrm{P}}\right) \mathcal{C}^{\mathrm{P}}[1]$. Here, however, we focus on the impact of the Peterlin approximation upon the statistics of polymer deformation and consider passive polymers only (one-way coupling). In the rest of the paper, we thus study the relation between Eq. (8) and Eq. (1) when $\sigma(t)$ is given by the incompressible Navier-Stokes equations in the turbulent regime. We expect the Peterlin approximation to be inaccurate when the velocity gradient is much larger than the polymer relaxation time, because in this case the distribution of polymer extensions is broad [43] and hence the factor $\phi\left(R^{2}\right)$ exhibits large fluctuations.

We end this section with some considerations that will be useful to guide our study:

(i) $\boldsymbol{R}(t)$ cannot be calculated from the solution of the FENE$\mathrm{P}$ equation [Eq. (8)]. By contrast, $\mathcal{C}(t)$ can be constructed from the solution of the FENE equation [Eq. (1)] by averaging the dyadic $R_{i}(t) R_{j}(t)$ over the realizations of the noise $\boldsymbol{\xi}(t)$. The tensor $\mathcal{C}(t)$ can then be compared with $\mathcal{C}^{\mathrm{P}}(t)$.

(ii) Because of the Peterlin approximation [Eq. (6)], the FENE and FENE-P equations yield a different evolution for the conformation tensor. This holds for both laminar and turbulent flows.

(iii) If the flow is turbulent, the statistics of $\boldsymbol{R}(t)$ differs from that of $\mathcal{C}(t)$, even if $\mathcal{C}(t)$ is calculated from Eq. (1) (and hence no closure approximation is required). Consider, for example, the random variables $R(t)$ and $\rho(t) \equiv \sqrt{\left\langle R^{2}(t)\right\rangle_{\xi}}=\sqrt{\operatorname{tr} \mathcal{C}(t)}$. In general, the probability density function (PDF) of $R(t)$ is different from that of $\rho(t)$, as can be seen from $\left\langle\left\langle R^{\alpha}\right\rangle_{\xi}\right\rangle_{\sigma} \neq$ $\left\langle\left\langle R^{2}\right\rangle_{\xi}^{\frac{\alpha}{2}}\right\rangle_{\sigma}(\alpha \neq 2)$, where $\langle\cdot\rangle_{\sigma}$ denotes an average over the statistics of the turbulent velocity gradient.

In conclusion, the FENE and FENE-P models differ for two reasons, namely the Peterlin approximation and the statistical effect due to the fact that the statistics of $\boldsymbol{R}(t)$ cannot be deduced from that of $\mathcal{C}(t)$. Hence, in the turbulent regime, the proper way to examine the Peterlin approximation is to first construct $\mathcal{C}(t)$ from the FENE equation and then compare its statistics with that of the solution of the FENE-P equation. If the statistics of $\boldsymbol{R}(t)$ is directly compared with that of $\mathcal{C}^{\mathrm{P}}(t)$, the error due to the Peterlin approximation is combined with the statistical effect discussed at point (iii) above. This fact seems to have been overlooked in some of the previous studies. We also remark that for the above reasons, our study differs from that presented in Ref. [15]. In that latter study, the statistics of $\boldsymbol{R}$ was indeed compared with that of the end-to-end separation vector of the FENE-P dumbbell model.

\section{LAGRANGIAN SIMULATIONS}

The dynamics of polymers is studied by using a database of Lagrangian trajectories that was previously generated to examine the dynamics of both tracers and inertial particles in turbulent flows $[44,45]$. The turbulent velocity field is obtained by direct numerical simulation of the three-dimensional incompressible Navier-Stokes equations:

$$
\partial_{t} \boldsymbol{u}+\boldsymbol{u} \cdot \nabla \boldsymbol{u}=-\nabla p+v \nabla^{2} \boldsymbol{u}+\boldsymbol{f}, \quad \nabla \cdot \boldsymbol{u}=0,
$$

where $p$ is the pressure field and $v=2 \times 10^{-3}$ is the kinematic viscosity. The forcing $f$ is such that the spectral content of the first low-wave-number shells remains constant in time. The domain is a three-dimensional periodic box of linear size $2 \pi$. Equation (9) are solved by means of a fully dealiased pseudospectral algorithm with second-order Adams-Bashforth time stepping. The number of grid points is $512^{3}$, while the integration time step is $d t=4 \times 10^{-4}$. In this simulation, the Kolmogorov time is $\tau_{\eta}=4.7 \times 10^{-2}$ and the Taylormicroscale Reynolds number is $R_{\lambda}=185$ (for more details on the numerical simulation, see Refs. [44,45]). We expect our results not to depend significantly on the value of $R_{\lambda}$ except for some residual effects induced by intermittency in the statistics of the velocity gradients [46].

As mentioned in Sec. II, the inertia of polymers is negligible. Furthermore, their thermal diffusivity is very small compared to the turbulent diffusivity. Hence the center of mass of a polymer moves like a tracer and its position $\boldsymbol{x}_{c}(t)$ satisfies the following equation:

$$
\frac{d \boldsymbol{x}_{c}}{d t}=\boldsymbol{u}\left(\boldsymbol{x}_{c}(t), t\right)
$$

Equation (10) is once again solved by using a second-order Adams-Bashforth scheme; a trilinear interpolation algorithm is used to determine the value of the velocity field at the position of the polymer $[44,45]$. After the statistically stationary state is reached for both the fluid motion and the translational dynamics of polymers, the positions of the center of mass of $128 \times 10^{3}$ polymers are recorded every $\Delta t=10 \mathrm{~d} t=4 \times 10^{-3} \approx \tau_{\eta} / 10$. The total integration time after steady state is $T=13.2$, which corresponds to six eddy turnover times approximately.

The velocity gradient $\sigma(t)$ is recorded every $\Delta t$ along the trajectory of the center of mass of each polymer and is inserted into Eqs. (1) and (8) in order to determine the dynamics of the separation vector and of the conformation tensor. Equation (1) is solved by using the semi-implicit predictor-corrector method introduced by Öttinger [42]; the integration time step is equal to $\Delta t$ for all values of the parameters. The initial condition for Eq. (1) is such that $R_{i}(0)=R_{0} / \sqrt{3}, i=1,2,3$. Equation (8) for $\mathcal{C}^{\mathrm{P}}$ is integrated by means of the semi-implicit algorithm proposed in Ref. [10], which ensures that $\operatorname{tr} \mathcal{C}^{\mathrm{P}} \leq L^{2}$.

The Weissenberg number is defined as $\mathrm{Wi} \equiv \tau_{p} / \tau_{\eta}$ and is the ratio of the time scales associated with the elastic 

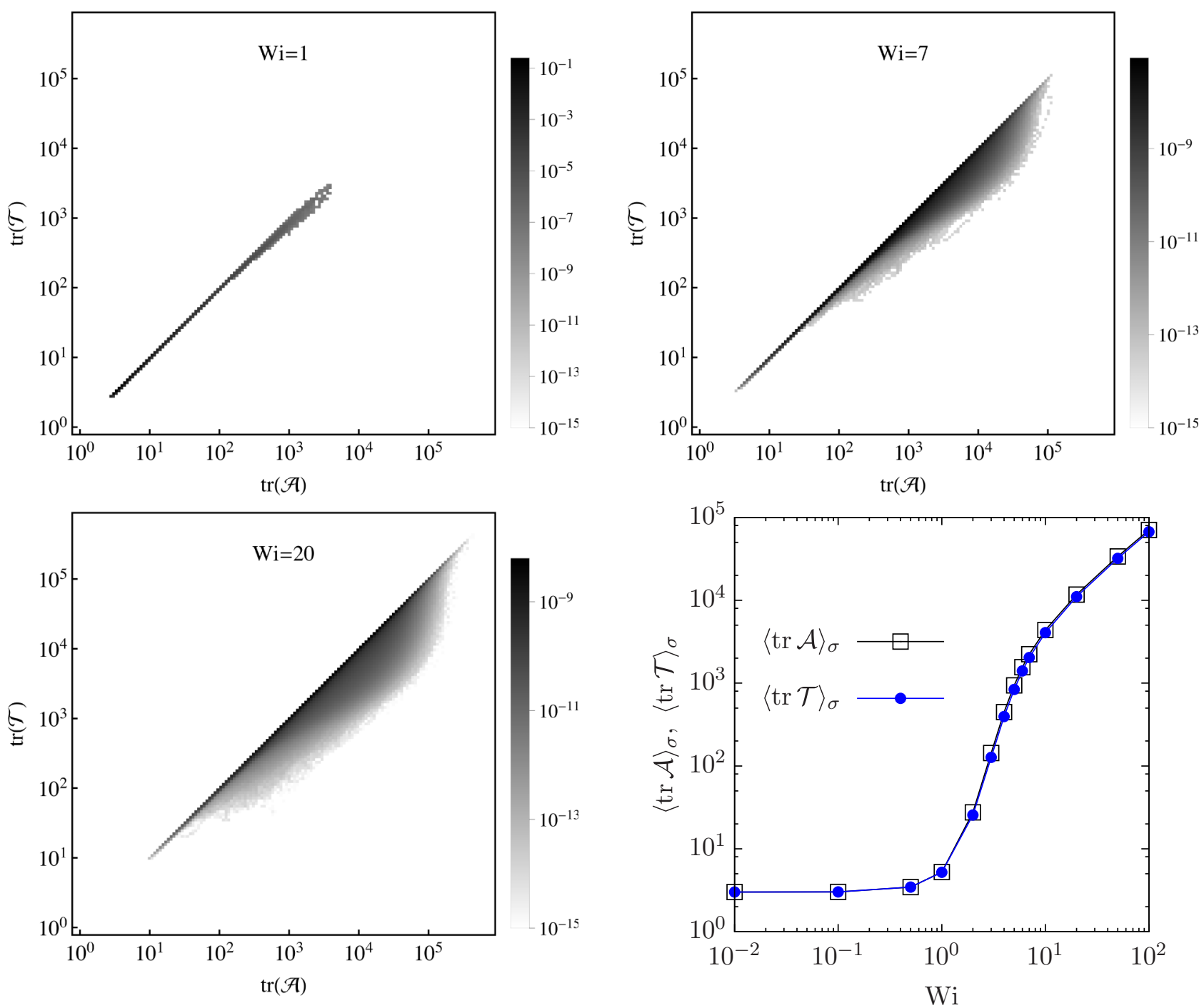

FIG. 1. (Color online) Contour plot of $P_{\sigma}(\operatorname{tr} \mathcal{A}, \operatorname{tr} \mathcal{T})$ for $\mathrm{Wi}=1$ (top left panel), Wi $=7$ (top right panel), and Wi $=20$ (bottom left panel). The bottom right panel shows the averages of $\operatorname{tr} \mathcal{A}$ (black squares) and $\operatorname{tr} \mathcal{T}$ (blue circles) as a function of the Weissenberg number.

force and with the velocity gradient. In our simulations, Wi varies between $10^{-2}$ and $10^{2}$. (An alternative definition of the Weissenberg number uses the maximum Lyapunov exponent of the flow, $\lambda$, to estimate the reciprocal of the stretching time associated with the velocity gradient. In our simulations, $\lambda \approx 0.14 \tau_{\eta}^{-1}$ [47]. Thus, the Weissenberg number based on the Lyapunov exponent is $\mathrm{Wi}_{\lambda}=\lambda \tau_{p} \approx 0.14 \mathrm{Wi}$.) The squares of the equilibrium and maximum extensions of the polymer are $R_{0}^{2}=1$ and $L^{2}=3 \times 10^{3}$, as in Refs. [9,19]. The number of realizations of thermal noise per Lagrangian trajectory is $2 \times 10^{3}$.

Finally, the statistics of polymer deformation is collected over the Lagrangian trajectories, over the realizations of thermal noise, and over time [only for times greater than the time required for $\boldsymbol{R}(t)$ to reach the statistically steady state].

We note that the statistics of the separation vector $\boldsymbol{R}$ in isotropic turbulence has been studied thoroughly by Watanabe and Gotoh [9]. The results on the statistics of $\boldsymbol{R}$ given below agree with those presented in Ref. [9]. Here, we compare the statistics of $\mathcal{C}$ with that of $\mathcal{C}^{\mathrm{P}}$ in order to determine the effect of the Peterlin closure on the dynamics of polymers.

\section{RESULTS}

In this section, we examine the statistics of polymer extension and orientation in the FENE and FENE-P models. Before presenting the results, it is useful to define some notations. If the statistics of a random variable depends both on thermal noise and on the velocity gradient (such as, for instance, in the case of $R$ ), its PDF is denoted as $P_{\xi, \sigma}$. If the statistics of a random variable (e.g., $\operatorname{tr} \mathcal{C}$ ) only depends on the velocity gradient, then its PDF is denoted as $P_{\sigma}$. The autocorrelation function of a scalar random variable $X(t)$ is denoted as $F_{X}(t)$ and the correlation time of $X(t)$ is $T_{X}=$ $\int_{0}^{\infty} F_{X}(t) d t$. The autocorrelation function of a statistically isotropic unit random vector $\boldsymbol{X}(t)$ is defined as

$$
F_{\boldsymbol{X}}(t)=2\left\langle\left|\boldsymbol{X}\left(t+t_{0}\right) \cdot \boldsymbol{X}\left(t_{0}\right)\right|\right\rangle-1
$$

and the associated correlation time is $T_{X}=\int_{0}^{\infty} F_{X}(t) d t$. We denote by $\hat{\boldsymbol{e}}_{i}(i=1,2,3)$ the unit eigenvectors of the rateof-strain tensor $\mathcal{S} \equiv\left(\nabla \boldsymbol{u}+\nabla \boldsymbol{u}^{\mathrm{T}}\right) / 2$; the eigenvectors $\hat{\boldsymbol{e}}_{i}$ are ordered by descending eigenvalue, i.e., $\hat{\boldsymbol{e}}_{1}$ is associated with the largest eigenvalue of $\mathcal{S}$ and $\hat{\boldsymbol{e}}_{3}$ with the smallest one. The 

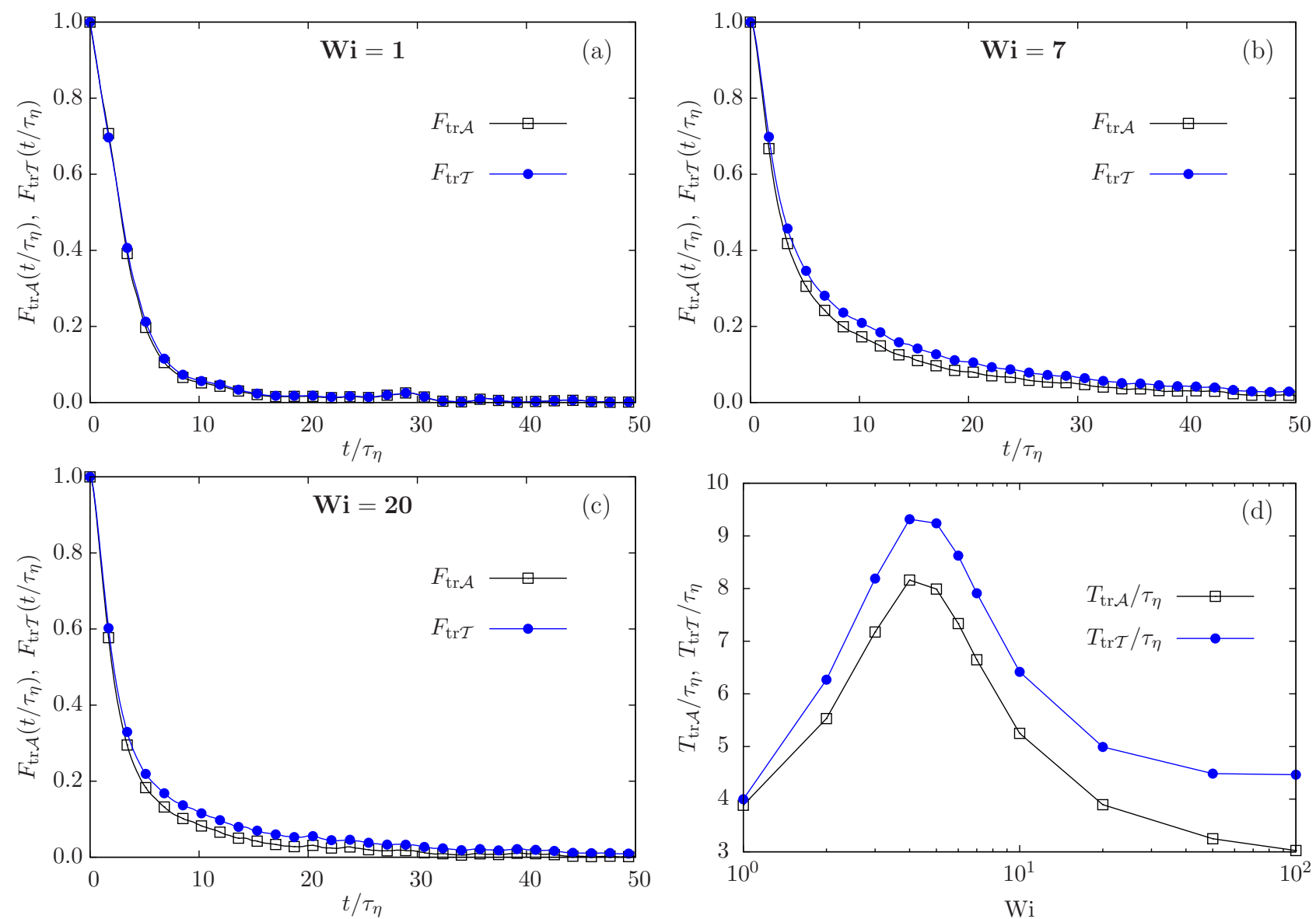

FIG. 2. (Color online) Autocorrelation function of $\operatorname{tr} \mathcal{A}$ (black squares) and $\operatorname{tr} \mathcal{T}$ (blue circles) for (a) Wi $=1$, (b) $\mathrm{Wi}=7$, and (c) Wi $=20$. Panel (d) shows the correlation times $T_{\text {tr } \mathcal{A}}$ (black squares) and $T_{\text {tr } \mathcal{T}}$ (blue circles) rescaled by $\tau_{\eta}$ as a function of Wi.

direction of vorticity is $\hat{\boldsymbol{\omega}}$. Finally, we denote by $\hat{z}_{1}$ and $\hat{z}_{1}^{\mathrm{P}}$ the first unit eigenvector of $\mathcal{C}$ and $\mathcal{C}^{\mathrm{P}}$, respectively.

\section{A. The Peterlin approximation}

As mentioned in Sec. II, the Peterlin approximation consists in replacing $\mathcal{A}$ with $\mathcal{T}$ in the evolution equation for the polymer conformation tensor $[\mathcal{A}$ and $\mathcal{T}$ have been defined in Eqs. (5) and (7)]. The eigenvectors of $\mathcal{A}$ and $\mathcal{T}$ are the same, but their eigenvalues may differ. Indeed, Jensen's inequality yields $\operatorname{tr} \mathcal{A} \geq \operatorname{tr} \mathcal{T}$. A first indication of the effect of the Peterlin approximation is thus given by the joint $\operatorname{PDF} P_{\sigma}(\operatorname{tr} \mathcal{A}, \operatorname{tr} \mathcal{T})$ (see Fig. 1). For small polymer extensions or for small values of $\mathrm{Wi}, \operatorname{tr} \mathcal{A}$ and $\operatorname{tr} \mathcal{T}$ are approximately the same, i.e., the Peterlin approximation holds very well. This fact can be explained by noting that, for small Wi, polymers are weakly stretched and hence $1-R^{2} / L^{2} \approx 1$. By contrast, for large extensions or for large values of Wi, the deviations of $\operatorname{tr} \mathcal{T}$ from $\operatorname{tr} \mathcal{A}$ are appreciable, i.e., the Peterlin approximation is inaccurate. As mentioned earlier, these deviations are due to the broad distribution of polymer extensions in a turbulent flow at large $\mathrm{Wi}$ (see Sec. IV B for more details). Nevertheless, $\langle\operatorname{tr} \mathcal{T}\rangle_{\sigma}$ and $\langle\operatorname{tr} \mathcal{A}\rangle_{\sigma}$ do not differ appreciably (see the bottom-right panel in Fig. 1). This fact demonstrates that the study of average values may not suffice to investigate the validity of the Peterlin approximation and that the fluctuations of the restoring term should also be analyzed, as they may influence the statistics of polymer deformation in a nontrivial way.

In addition, the qualitative behavior of the temporal autocorrelations of $\operatorname{tr} \mathcal{A}$ and $\operatorname{tr} \mathcal{T}$ are similar [Figs. 2(a)-2(c)], but for large Wi the correlation time of $\operatorname{tr} \mathcal{A}$ is shorter than that of $\operatorname{tr} \mathcal{T}$ [Fig. 2(d)]. The peak in the graph of the correlation time versus Wi can be attributed to the critical slowing down of polymer stretching near the coil-stretch transition [17,48,49] (see also Sec. IV B).

\section{B. Statistics of polymer extension}

The statistical properties of the separation $R$ are well understood; we briefly review the theory of the coil-stretch transition, which will be useful to interpret the numerical simulations. For small values of Wi, most of the polymers are in the coiled state, i.e., their extension is close to the equilibrium one. Accordingly, the PDF of $R$ has a pronounced peak at $R_{0}$. As Wi increases, polymers unravel and become more and more extended. The transition from the coiled to the stretched state occurs when the Lyapunov exponent of the flow exceeds $1 / 2 \tau_{p}$, i.e., at $\mathrm{Wi}_{\lambda}=1 / 2$ [43]. [Note that some authors define the Weissenberg number in terms of the time scale associated with the exponential relaxation of $\sqrt{\left\langle R^{2}(t)\right\rangle}$ instead of $\left\langle R^{2}(t)\right\rangle$ and hence obtain a critical Weissenberg number equal to unity.] At intermediate extensions, the PDF 

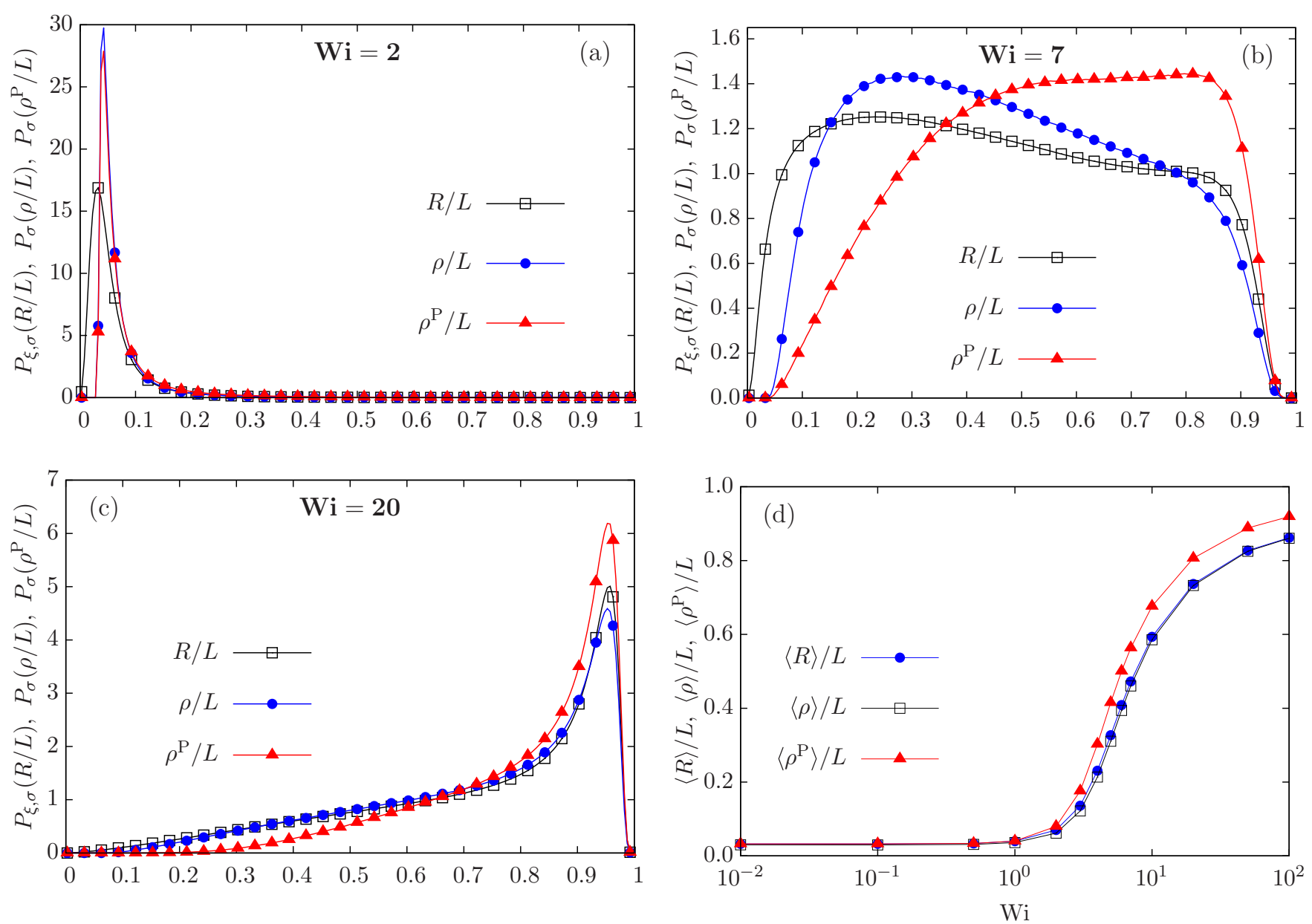

FIG. 3. (Color online) PDF of $R / L$ (black squares), $\rho / L$ (blue circles), and $\rho^{\mathrm{P}} / L$ (red triangles) for (a) $\mathrm{Wi}=2$, (b) $\mathrm{Wi}=7$, and (c) $\mathrm{Wi}=20$. Panel (d) shows $\langle R\rangle_{\xi, \sigma} / L$ (black squares), $\langle\rho\rangle_{\sigma} / L$ (blue circles), and $\left\langle\rho^{\mathrm{P}}\right\rangle_{\sigma} / L$ (red triangles) as a function of the Weissenberg number.

of $R$ has a power-law behavior, i.e., $P_{\xi, \sigma}(R) \sim R^{-1-\alpha}$ for $R_{0} \ll R \ll L$ [43]. This property of $P_{\xi, \sigma}(R)$ indicates that polymers with very different extensions coexist in the fluid; whether the coiled or the stretched state dominates depends on the value of $\mathrm{Wi}$. The exponent $\alpha$ is positive in the coiled state and decreases as a function of Wi $[9,20,43,50]$. As long as $\alpha>0$, the FENE dumbbell model reaches a steady state even as $L$ tends to infinity (the $L \rightarrow \infty$ limit of the FENE model is known as the Hookean model [1]). However, when $\alpha$ vanishes, a steady-state PDF of the extension no longer exists if $L \rightarrow \infty$. This behavior is interpreted as the coil-stretch transition [43]. Finally, if Wi increases beyond the value of the coil-stretch transition, $\alpha$ becomes negative and the maximum of $P_{\xi, \sigma}(R)$ moves from close to $R_{0}$ to close to $L[9,50]$. The statistics of $R$ shown in Figs. 3 and 4 for different values of Wi agrees very well with the theory. We now compare the FENE and FENE-P models and use the above theoretical predictions to interpret the numerical results.

We noted in Sec. II that the comparison between the FENE and the FENE-P models ought to be done in terms of the conformation tensors $\mathcal{C}$ and $\mathcal{C}^{\mathrm{P}}$ (rather than in terms of $R$ and $\left.\mathcal{C}^{\mathrm{P}}\right)$. Let us denote $\rho(t)=\sqrt{\operatorname{tr} \mathcal{C}(t)}$ and $\rho^{\mathrm{P}}(t)=\sqrt{\operatorname{tr} \mathcal{C}^{\mathrm{P}}(t)}$. To examine the influence of the Peterlin approximation on the statistics of polymer extension, we calculate $\rho$ from the solution of Eq. (1) and $\rho^{\mathrm{P}}$ from Eq. (8). We then compare $P_{\sigma}(\rho / L)$ and $P_{\sigma}\left(\rho^{\mathrm{P}} / L\right)$ in the steady state for different values of Wi. The plots shown in Figs. 3 and 4 correspond to the coiled state $(\mathrm{Wi}=2)$, the coil-stretch transition $(\mathrm{Wi}=7)$, and the stretched state $(\mathrm{Wi}=20)$.

For small values of Wi, $P_{\sigma}(\rho / L)$ and $P_{\sigma}\left(\rho^{\mathrm{P}} / L\right)$ do not differ significantly. The reason for this behavior is that for small Wi, the extension of most polymers is near $R_{0}$; hence $1-R^{2} / L \approx$ 1 and the Peterlin approximation holds very well [Fig. 3(a)]. For intermediate and large values of $\mathrm{Wi}$, the differences between $P_{\sigma}(\rho / L)$ and $P_{\sigma}\left(\rho^{\mathrm{P}} / L\right)$ are, in contrast, considerable. This fact is due to the power-law behavior of the PDF of $R$ for intermediate extensions. At large $\mathrm{Wi}$, the statistics of $R$ is indeed characterized by a broad distribution of extensions around the mean value, and the Peterlin approximation is poor. In particular, we note that in the FENE-P model, large extensions are more probable than in the FENE model to the detriment of small and intermediate extensions [Figs. 3(b) and $3(\mathrm{c})$. This phenomenon is easily explained by noting that in the FENE model, the elastic force keeps $R^{2}$ smaller than $L^{2}$, whereas in the FENE-P model it only ensures that $\rho^{2}<L^{2}$. Hence the restoring term in Eq. (8) is weaker than that in 

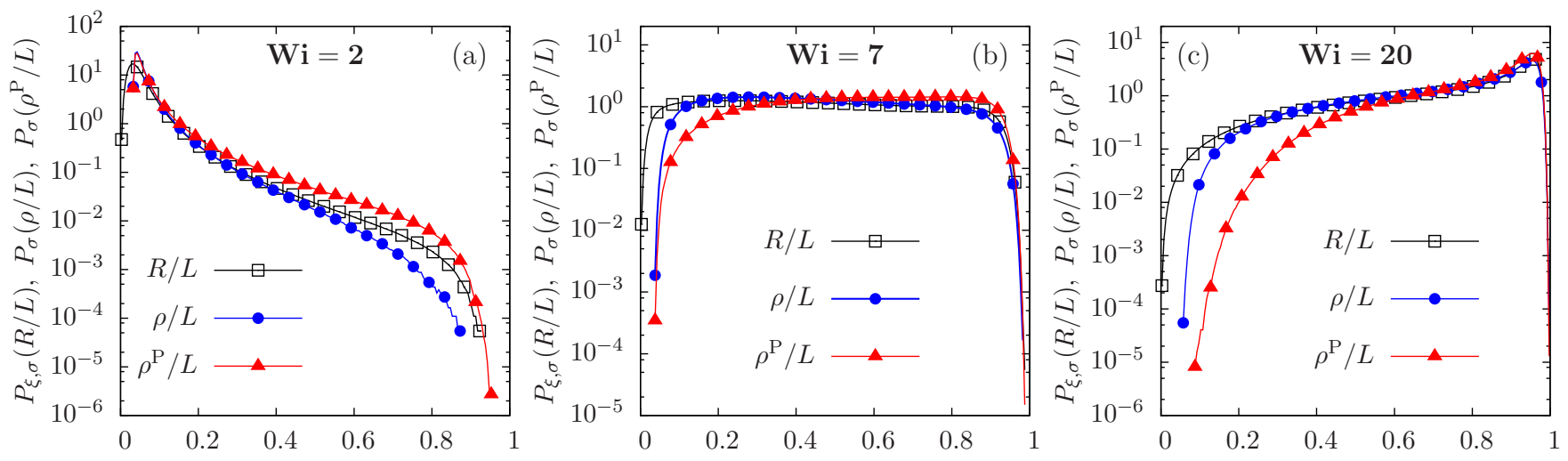

FIG. 4. (Color online) PDF of $R / L$ (black squares), $\rho / L$ (blue circles), and $\rho^{\mathrm{P}} / L$ (red triangles) in a semilogarithmic scale for $($ a) Wi $=2$, (b) $\mathrm{Wi}=7$, and (c) $\mathrm{Wi}=20$.

Eq. (1) and allows larger extensions. The FENE-P model, therefore, overestimates the average extension of polymers for large values of Wi [Fig. 3(d)]. An analogous behavior has been observed in turbulent channel flows $[10,15]$.

We also note that whereas for large Wi the PDFs of $R$ and $\rho$ are approximately the same [Fig. 3(c)], they are significantly different for intermediate or small Wi [Figs. 3(a) and 3(b)]. This fact can be explained as follows. In the former case, the stretching action of the velocity gradient is very strong compared to the effect of thermal fluctuations, and in most realizations $R \approx \sqrt{\operatorname{tr} \mathcal{C}}$. In the latter case, the effect of thermal fluctuations cannot be disregarded and the differences in the statistics of $R$ and $\rho$ (see Sec. II) become evident. Furthermore, $P_{\xi, \sigma}(R / L)$ and $P_{\sigma}(\rho / L)$ mainly differ for small
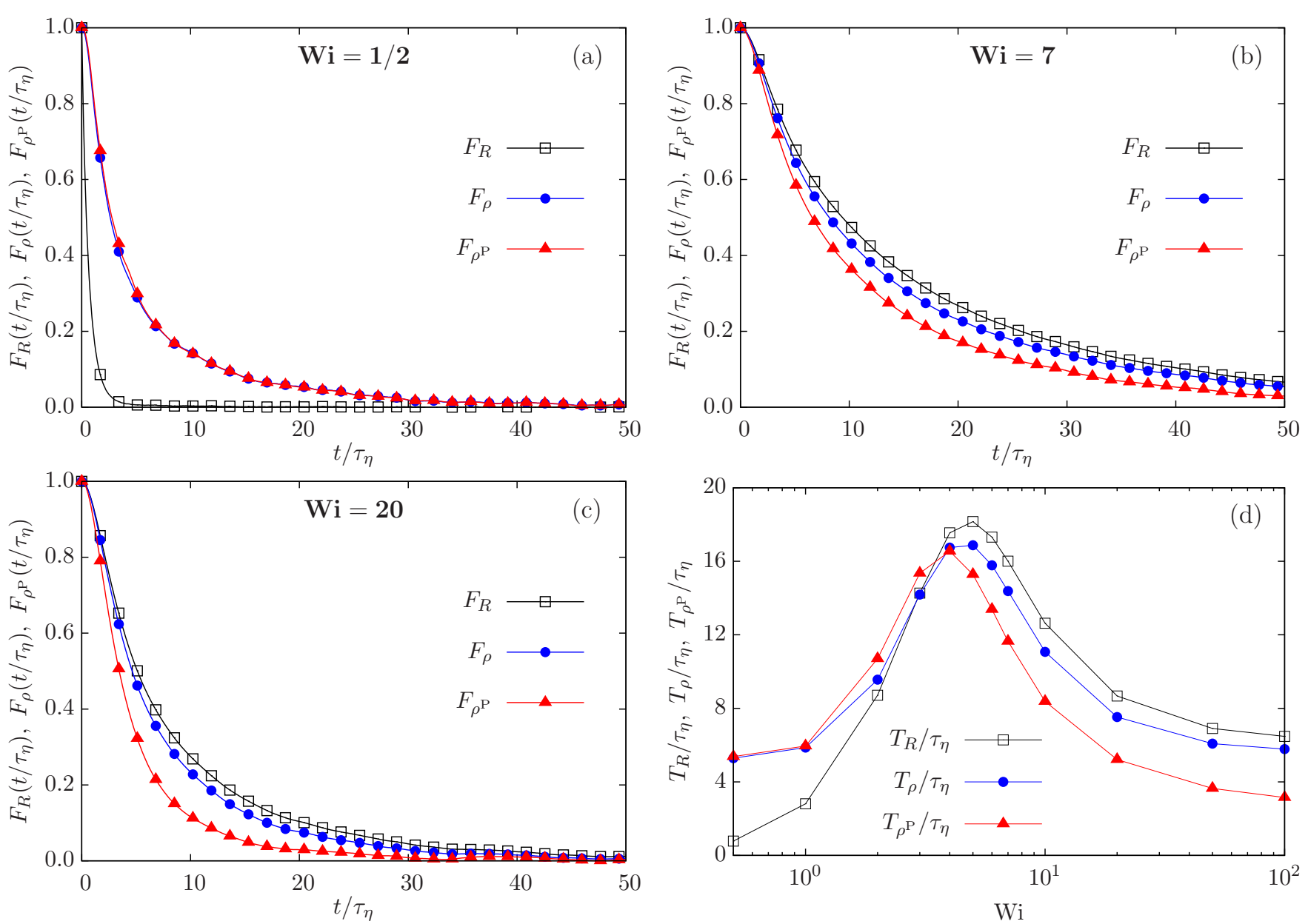

FIG. 5. (Color online) Autocorrelation function of $R$ (black squares), $\rho$ (blue circles), and $\rho^{\mathrm{P}}$ (red triangles) for (a) Wi $=0.5$, (b) Wi $=7$, and (c) $\mathrm{Wi}=20$. Panel (d) shows the correlation times $T_{R}$ (black squares), $T_{\rho}$ (blue circles), and $T_{\rho} \mathrm{P}$ (red triangles) rescaled by $\tau_{\eta}$ as a function of the Weissenberg number. 

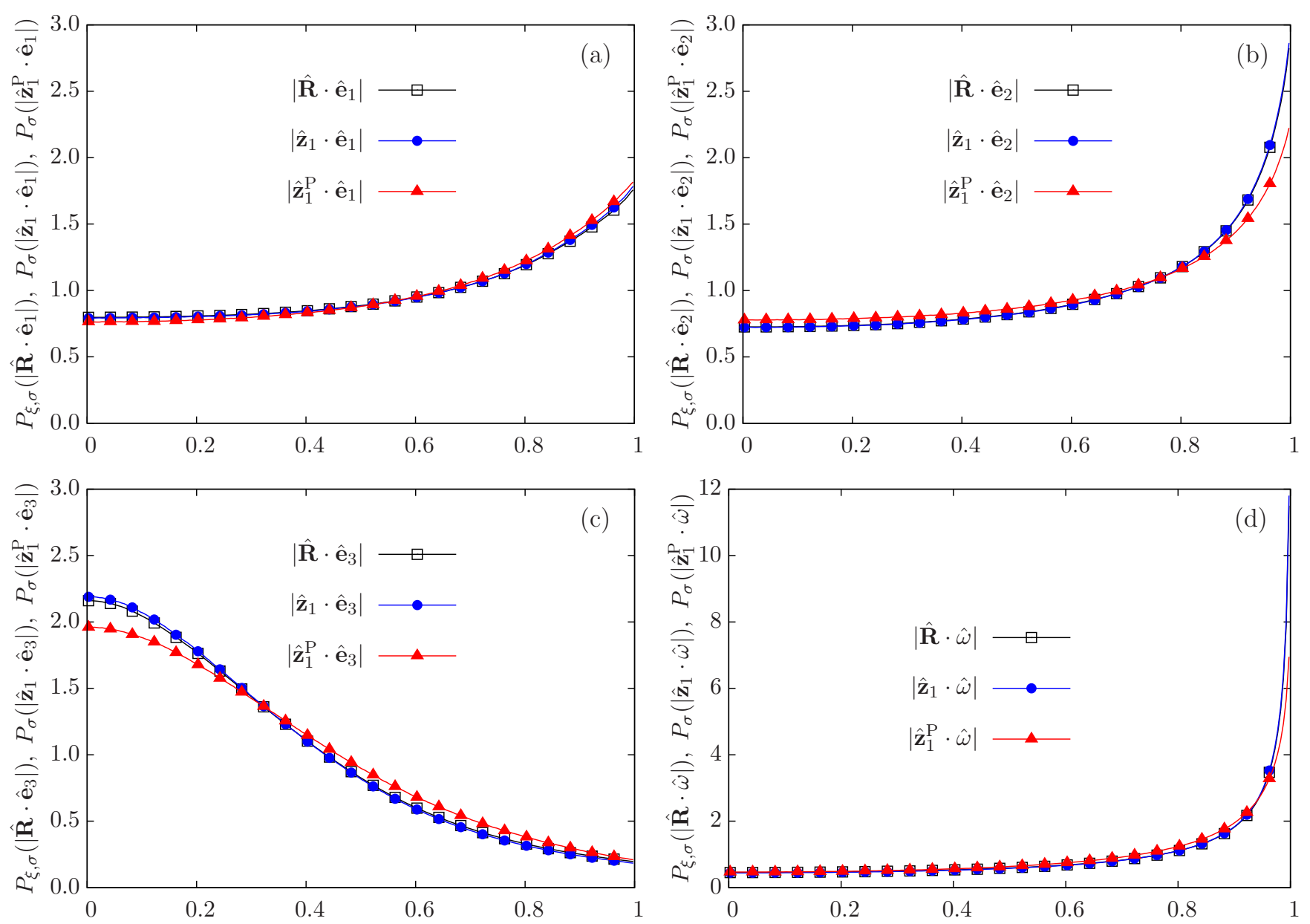

FIG. 6. (Color online) PDF of alignment with (a) $\hat{\boldsymbol{e}}_{1}$, (b) $\hat{\boldsymbol{e}}_{2}$, (c) $\hat{\boldsymbol{e}}_{3}$, and (d) $\hat{\boldsymbol{\omega}}$ for Wi $=20$.

and intermediate extensions, because the large extensions are obtained in those realizations in which the velocity gradient is very intense and thermal noise can be neglected. The above results demonstrate that comparing the statistics of $\boldsymbol{R}$ directly with that of $\mathcal{C}^{\mathrm{P}}$ may lead to wrong conclusions; indeed, at small Wi, $P_{\xi, \sigma}(R / L)$ and $P_{\sigma}\left(\rho^{\mathrm{P}} / L\right)$ are clearly different, whereas $P_{\sigma}(\rho / L)$ and $P_{\sigma}\left(\rho^{\mathrm{P}} / L\right)$ are close.

The autocorrelation function of the extension is approximately exponential both in the FENE and in the FENE-P models. However, $F_{\rho^{\mathrm{P}}}(t)$ is a good approximation of $F_{\rho}(t)$ only for small Wi [Fig. 5(a)]. In addition, the FENE-P model captures the critical slowing down of polymers near the coilstretch transition $[17,48,49]$, but for large Wi it underestimates the correlation time of the extension [Fig. 5(d)]. Once again, we note that, for small $\mathrm{Wi}$, a direct comparison between $F_{R}(t)$ and $F_{\rho} \mathrm{P}(t)$ would lead to wrong conclusions about the effect of the Peterlin closure on the temporal statistics of polymer extension.

\section{Statistics of polymer orientation}

In a coupled simulation of turbulent drag reduction, the feedback of polymers on the flow is of a tensorial nature; therefore, it depends not only on the extension of polymers but also on their orientation.
In the FENE and FENE-P models, the first eigenvector of $\mathcal{C}$ and of $\mathcal{C}^{\mathrm{P}}$ (denoted as $\hat{z}_{1}$ and $\hat{\boldsymbol{z}}_{1}^{\mathrm{P}}$, respectively) gives the direction of the polymer, provided Wi is sufficiently large. The effect of the Peterlin approximation on polymer orientation can then be studied by comparing the statistics of $\hat{z}_{1}^{\mathrm{P}}$ with that of $\hat{z}_{1}$.

Figure 6 shows that $\hat{z}_{1}$ exhibits a moderate alignment with $\hat{\boldsymbol{e}}_{2}$ and a strong alignment with $\hat{\boldsymbol{\omega}}$. This behavior can be explained as follows. We have already noted that, for intermediate or large values of $\mathrm{Wi}$, thermal fluctuations have a negligible effect on the dynamics of polymers. We therefore expect that the statistics of $\hat{\boldsymbol{z}}_{1}$ coincides with that of $\hat{\boldsymbol{R}}=\boldsymbol{R} / R$, as is confirmed by the results shown in Fig. 6. If thermal fluctuations are negligible, the evolution of the extension $R$ decouples from that of the orientation $\hat{\boldsymbol{R}}$, which behaves like the orientation vector of a rigid rod and is the solution of the Jeffery equation [51] [see also Fig. 7(b) in Ref. [19]]. Therefore, for large values of $\mathrm{Wi}$, the orientation statistics of $\hat{z}_{1}$ can be deduced from the dynamics of rigid rods. Pumir and Wilkinson [52] have shown that in isotropic turbulence there is a moderate alignment between rigid rods and $\hat{\boldsymbol{e}}_{2}$, whereas the alignment of rods with the direction of vorticity is very strong. This phenomenon explains the alignment properties of $\hat{z}_{1}$ (Fig. 6). Figure 6 also compares the statistics of $\hat{z}_{1}$ and $\hat{z}_{1}^{\mathrm{P}}$. The FENE-P model qualitatively reproduces the orientation statistics of the first eigenvector of the 

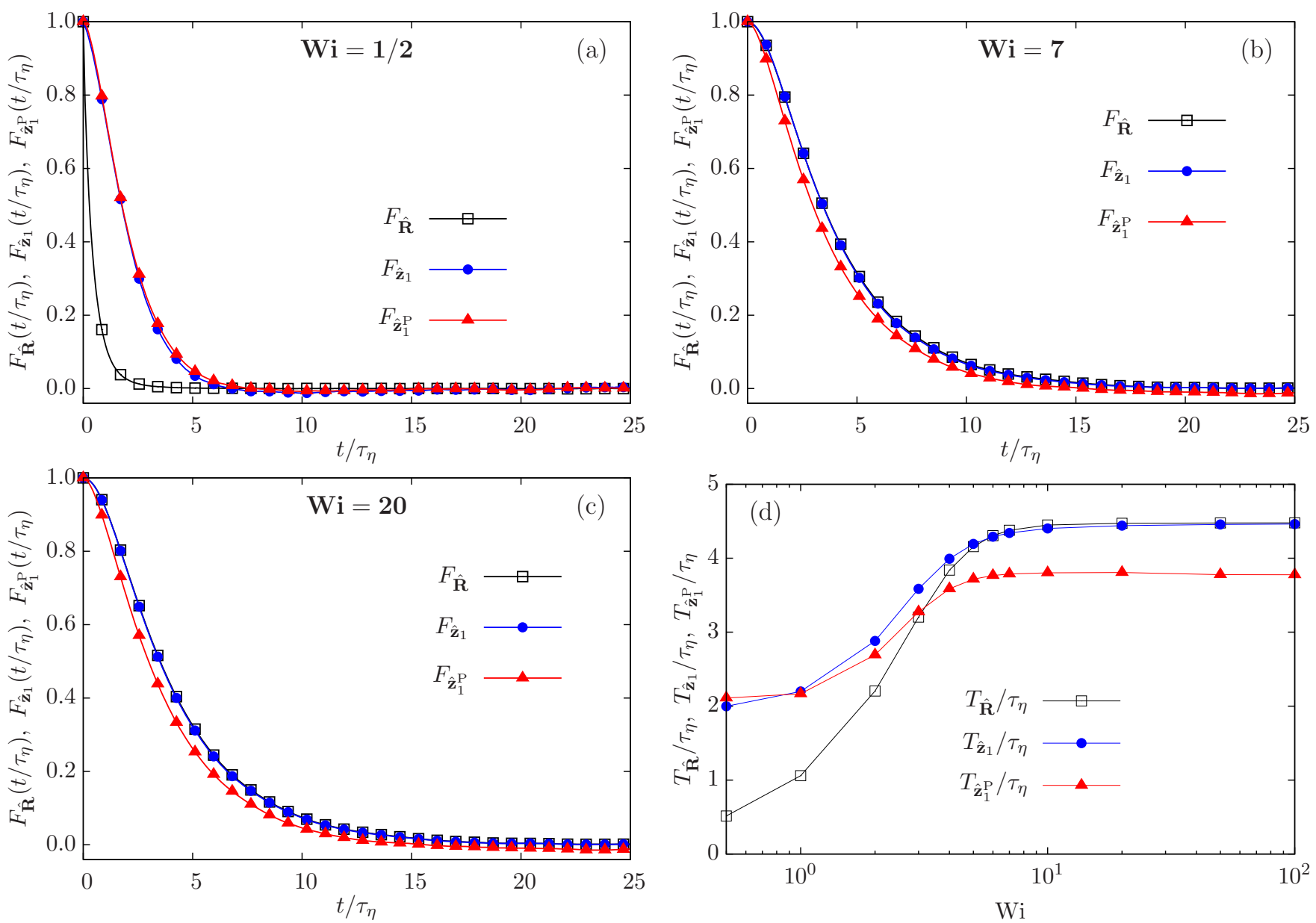

FIG. 7. (Color online) Autocorrelation function of $\hat{\boldsymbol{R}}$ (black squares), $\hat{\boldsymbol{z}}_{1}$ (blue circles), and $\hat{\boldsymbol{z}}_{1}^{\mathrm{P}}$ (red triangles) for $(\mathrm{a}) \mathrm{Wi}=0.5$, (b) Wi $=7$, and (c) $\mathrm{Wi}=20$. Panel (d) shows the correlation times $T_{\hat{\boldsymbol{R}}}$ (black squares), $T_{\hat{z}_{1}}$ (blue circles), and $T_{\hat{z}_{1}^{\mathrm{P}}}$ (red triangles) rescaled by $\tau_{\eta}$ as a function of the Weissenberg number.

conformation tensor but underestimates the level of alignment (Fig. 6).

The autocorrelation function of $\hat{\boldsymbol{z}}_{1}$ decays exponentially [Figs. 7(a)-7(c)], which for large Wi is once again in agreement with the results for the autocorrelation of the orientation of a rod [52]. The correlation time of $\hat{z}_{1}$ increases for small values of Wi and saturates at large Wi [Fig. 7(d)]. The saturation is due to the fact that at large Wi the statistics of $\hat{\boldsymbol{R}}$ and $\hat{\boldsymbol{z}}_{1}$ are the same and the evolutions of $R$ and $\hat{\boldsymbol{R}}$ decouple [51], which implies that the statistics of $\hat{\boldsymbol{R}}$ agrees with the orientation statistics of a rod and therefore becomes independent of $\mathrm{Wi}$. The results of the FENE-P model are in agreement with these properties of the FENE model at small Wi, but quantitative discrepancies appear at large Wi [Figs. 7(a) to 7(c)]. In particular, the FENE-P model underestimates the correlation time of polymer orientation [Fig. 7(d)].

\section{CONCLUSIONS}

Numerical simulations of turbulent flows of polymer solutions use the FENE-P model, which is based on the elastic dumbbell model but requires a closure approximation for the elastic term. We have examined the effect of the Peterlin closure on the steady-state statistics of the extension and the orientation of polymers. The FENE-P model captures the qualitative properties of the statistics, but for large Wi it underestimates the steady-state probability of small extensions and overestimates the probability of large extensions. As a consequence, the Peterlin approximation yields a greater average extension as well as a greater probability that polymers break under the action of a turbulent flow [53]. To quantify this effect, one would need to couple the dynamics of polymers with a fragmentation model connected to the accumulated (or instantaneous) stress along each trajectory and to estimate the relative breaking rate [54]. Since large polymer extensions are more likely in the FENE-P model than in the FENE model, we also expect that the Peterlin approximation yields a stronger feedback of polymers on the flow in two-way coupling simulations of homogeneous isotropic turbulence with polymer additives. A similar argument, however, does not carry over to inhomogeneous flows such as channel flows. In this case, indeed, drag reduction is caused by the strong stretching of polymers in the near-wall region rather than by the dynamics in the bulk of the channel, where the flow is homogeneous and isotropic, and a lower degree of stretching is observed [2,10,12-14,21].

As regards the temporal statistics of the end-to-end separation vector, both the correlation times of the extension 
and the orientation of polymers are underestimated by the FENE-P model. The FENE-P model also underestimates the level of alignment of polymers with the eigenvectors of the rate-of-strain tensor and with the direction of vorticity.

It would be interesting to check to what extent these properties of the FENE-P model influence the dynamics of a polymer solution by comparing two-way coupling simulations of the FENE-P model in which the stress tensor is either calculated according to the Peterlin closure or from molecular dynamics.

In this paper, we examined the Peterlin approximation, as this is the main assumption in the construction of a continuum model of polymer solutions. However, it is worth recalling that the FENE-P model is based on the dumbbell model and hence on a very simplified coarse-grained description of a polymer macromolecule. Other approximations may thus impact the performance of the FENE-P model and its comparison with experiments. For instance, even for simple laminar flows, the dumbbell model reproduces the experimental observations only if the maximum extension and the effective bead radius are used as free parameters to fit the experimental data [55,56]. The FENE model also disregards hydrodynamical interactions between the beads of a single dumbbell. However, these hydrodynamical interactions only affect the statistics of small extensions and are negligible at large extensions, where discrepancies between the FENE and FENE-P model become appreciable. Thus, for sufficiently large $\mathrm{Wi}$, we expect the errors due to the absence of bead-bead hydrodynamical interactions to be subleading with respect to those due to the Peterlin closure. Finally, the FENE dumbbell model, which consists of a single oscillation mode, fails to accurately capture the deformation of a polymer after a sudden variation of the strain (see, e.g., Ref. [57]). To fix this limitation of the FENE model, Ghosh et al. [58] have derived a dumbbell model in which the maximum extension is adapted to the amplitude of the strain. This model was recently generalized by Gupta [59] to a bead-spring chain model that also describes the internal motions of a polymer molecule.

\section{ACKNOWLEDGMENTS}

This work was supported in part by the EU COST Action MP1305 "Flowing Matter" and by a research program of the Foundation for Fundamental Research on Matter (FOM), which is part of the Netherlands Organisation for Scientific Research (NWO). Part of the computations were done at "Mésocentre SIGAMM," Observatoire de la Côte d'Azur, Nice, France. D.V. acknowledges the hospitality of the Department of Physics of the Eindhoven University of Technology, where part of this work was done, and the Indo-French Centre for Applied Mathematics (IFCAM), Bangalore, for financial support.
[1] R. B. Bird, O. Hassager, R. C. Armstrong, and C. F. Curtiss, Dynamics of Polymeric Liquids (Wiley, New York, 1987), Vol. 2.

[2] I. Procaccia, V. S. L'Vov, and R. Benzi, Rev. Mod. Phys. 80, 225 (2008).

[3] C. M. White and M. G. Mungal, Annu. Rev. Fluid Mech. 40, 235 (2008).

[4] R. Pandit, P. Perlekar, and S. S. Ray, Pramana-J. Phys. 73, 157 (2009).

[5] R. Benzi, Physica D 239, 1338 (2010).

[6] M. D. Graham, Phys. Fluids 26, 101301 (2014).

[7] B. A. Toms, in Proceedings of the 1st International Congress on Rheology (North-Holland, Amsterdam, 1949), Vol. 2, pp. 135141.

[8] A. Gyr and H.-W. Bewersdorff, Drag Reduction of Turbulent Flows by Additives (Kluwer Academic, Dordrecht, 1995).

[9] T. Watanabe and T. Gotoh, Phys. Rev. E 81, 066301 (2010).

[10] V. E. Terrapon, Y. Dubief, P. Moin, and E. S. G. Shaqfeh, in Proceedings of ASME, ASME/JSME 4th Joint Fluids Engineering Conference (FEDSM2003), Honolulu, HI (ASME, 2003), pp. 773-780.

[11] H. Massah, K. Kontomaris, W. R. Schowalter, and T. J. Hanratty, Phys. Fluids A 5, 881 (1993).

[12] P. Ilg, E. De Angelis, I. V. Karlin, C. M. Casciola, and S. Succi, Europhys. Lett. 58, 616 (2002).

[13] Q. Zhou and R. Akhavan, J. Non-Newtonian Fluid Mech. 109, 115 (2003).

[14] V. E. Terrapon, Y. Dubief, P. Moin, E. S. G. Shaqfeh, and S. K. Lele, J. Fluid Mech. 504, 61 (2004).

[15] V. K. Gupta, R. Sureshkumar, and B. Khomami, Phys. Fluids 16, 1546 (2004).
[16] A. Celani, S. Musacchio, and D. Vincenzi, J. Stat. Phys. 118, 531 (2005).

[17] A. Celani, A. Puliafito, and D. Vincenzi, Phys. Rev. Lett. 97, 118301 (2006).

[18] J. Davoudi and J. Schumacher, Phys. Fluids 18, 025103 (2006).

[19] S. Jin and L. R. Collins, New J. Phys. 9, 360 (2007).

[20] S. Musacchio and D. Vincenzi, J. Fluid Mech. 670, 326 (2011).

[21] F. Bagheri, D. Mitra, P. Perlekar, and L. Brandt, Phys. Rev. E 86, 056314 (2012).

[22] M. Laso and H. C. Öttinger, J. Non-Newtonian Fluid Mech. 47, 1 (1993).

[23] K. Feigl, M. Laso, and H. C. Öttinger, Macromolecules 28, 3261 (1995).

[24] M. A. Hulsen, A. P. G. van Heel, and B. H. A. A. van den Brule, J. Non-Newtonian Fluid Mech. 70, 79 (1997).

[25] T. Peters and J. Schumacher, Phys. Fluids 19, 065109 (2007).

[26] T. Watanabe and T. Gotoh, J. Fluid Mech. 717, 535 (2013).

[27] T. Watanabe and T. Gotoh, Phys. Fluids 26, 035110 (2014).

[28] A. Peterlin, J. Polym. Sci. Pt. B: Polym. Lett. 4, 287 (1966).

[29] R. B. Bird, P. J. Dotson, and N. L. Johnson, J. Non-Newtonian Fluid Mech. 7, 213 (1980).

[30] R. Sureshkumar, A. N. Beris, and R. A. Handler, Phys. Fluids 9, 743 (1997).

[31] E. De Angelis, C. M. Casciola, and R. Piva, Comput. Fluids 31, 495 (2002).

[32] P. K. Ptasinski, B. J. Boersma, F. T. M. Nieuwstadt, M. A. Hulsen, B. H. A. A. Van den Brule, and J. C. R. Hunt, J. Fluid Mech. 490, 251 (2003).

[33] Y. Dubief, C. M. White, V. E. Terrapon, E. S. G. Shaqfeh, P. Moin, and S. K. Lele, J. Fluid Mech. 514, 271 (2004). 
[34] P. A. Stone, A. Roy, R. G. Larson, F. Waleffe, and M. D. Graham, Phys. Fluids 16, 3470 (2004).

[35] A. Robert, T. Vaithianathan, L. R. Collins, and J. G. Brasseur, J. Fluid Mech. 657, 189 (2010).

[36] E. De Angelis, C. M. Casciola, R. Benzi, and R. Piva, J. Fluid Mech. 531, 1 (2005).

[37] P. Perlekar, D. Mitra, and R. Pandit, Phys. Rev. Lett. 97, 264501 (2006).

[38] P. Perlekar, D. Mitra, and R. Pandit, Phys. Rev. E 82, 066313 (2010).

[39] A. Gupta, P. Perlekar, and R. Pandit, Phys. Rev. E 91, 033013 (2015).

[40] M. Herrchen and H. C. Öttinger, J. Non-Newtonian Fluid Mech. 68, 17 (1997).

[41] R. Keunings, J. Non-Newtonian Fluid Mech. 68, 85 (1997).

[42] H. C. Öttinger, Stochastic Processes in Polymeric Fluids (Springer, Berlin Heidelberg, 1996).

[43] E. Balkovsky, A. Fouxon, and V. Lebedev, Phys. Rev. Lett. 84, 4765 (2000).

[44] E. Calzavarini, M. Cencini, D. Lohse, and F. Toschi, Phys. Rev. Lett. 101, 084504 (2008).

[45] J. Bec, L. Biferale, A. Lanotte, A. Scagliarini, and F. Toschi, J. Fluid Mech. 645, 497 (2010).
[46] R. Benzi, L. Biferale, G. Paladin, A. Vulpiani, and M. Vergassola, Phys. Rev. Lett. 67, 2299 (1991).

[47] J. Bec, L. Biferale, G. Boffetta, M. Cencini, S. Musacchio, and F. Toschi, Phys. Fluids 18, 091702 (2006).

[48] D. Vincenzi and E. Bodenschatz, J. Phys. A 39, 10691 (2006).

[49] S. Gerashchenko and V. Steinberg, Phys. Rev. E 78, 040801 (2008).

[50] M. Martins Afonso and D. Vincenzi, J. Fluid Mech. 540, 99 (2005).

[51] W. L. Olbricht, J. M. Rallison, and L. G. Leal, J. Non-Newtonian Fluid Mech. 10, 291 (1982).

[52] A. Pumir and M. Wilkinson, New J. Phys. 13, 093030 (2011).

[53] J.-L. Thiffeault, Phys. Lett. A 308, 445 (2003).

[54] M. U. Babler, L. Biferale, and A. S. Lanotte, Phys. Rev. E 85, 025301(R) (2012).

[55] T. T. Perkins, D. E. Smith, and S. Chu, Science 276, 2016 (1997).

[56] R. G. Larson, T. T. Perkins, D. E. Smith, and S. Chu, Phys. Rev. E 55, 1794 (1997).

[57] I. Ghosh, G. H. McKinley, R. A. Brown, and R. C. Armstrong, J. Rheol. 45, 721 (2001).

[58] I. Ghosh, Y. L. Joo, G. H. McKinley, R. A. Brown, and R. C. Armstrong, J. Rheol. 46, 1057 (2002).

[59] V. K. Gupta, Rheol. Acta 51, 51 (2012). 